\title{
Éditorial : Promotion, politique et pratique
}

Je reviens d'un séjour de trois jours au Sheraton Centre de Toronto, où le CJA avait une table au Festival de conférences internationales relatives aux prestations de soins, aux incapacités, au vieillissement et à la technologie (FICCDAT). Le FICCDAT est un regroupement de cinq conférences qui ont eu lieu simultanément et attiré quelque 1200 participants de partout dans le monde. Au début de l'année, lorsque j'ai décidé que la CJA devrait avoir une table au FICCDAT, je voyais le festival comme une occasion de promouvoir la revue et par le fait même le CAG à un auditoire international $\mathrm{d}$ 'auteurs et de lecteurs potentiels. C'est en réalité la seconde fois que j'ai fait cela pendant mon mandat à titre de rédacteur en chef. Nous avions fait un effort similaire au VIII ${ }^{\mathrm{e}}$ Congrès international francophone de gérontologie et de gériatrie à Québec en octobre 2006. J'aurais aimé dire que notre table était submergée de délégués intéressés des deux conférences. Bien que le nombre de membres ait été stable à Québec et que notre expérience était pratiquement la même qu'à Toronto, le nombre de participants était loin d'être probant.

Quelles leçons le CJA et le CAG peuvent-ils tirer de ces deux expériences? Premièrement, même si le nombre de personnes qui ont visité notre table était relativement faible comparativement au nombre total de participants aux deux conférences, tisser de nouveaux liens avec des auteurs et des abonnés possibles, particulièrement avec ceux de l'extérieur du Canada, en vaut certainement la peine. Deuxièmement, il est aussi frappant que bon nombre des personnes qui sont venues me parler à notre table connaissaient très peu ou pas du tout le CJA ou le CAG. Cette seconde question me fait me demander de quelle façon le CJA et le CAG pourraient faire leur propre promotion à un moment où la concurrence pour des auteurs, des abonnés, des membres, et des fonds est de plus en plus serrée. Dans le contexte du mandat du CJA de publier les résultats de la recherche en gérontologie de la plus haute qualité jugée par les pairs, j'apprécierais certainement recevoir vos suggestions. Troisièmement, je crois que tous les membres du CAG doivent devenir des ambassadeurs du CJA et du CAG et inciter leurs pairs à présenter leurs meilleurs travaux au $C J A$ et à participer à d'autres activités du CAG. Cela vise à suggérer non pas que nous devrions cesser d'être constructivement critique $\mathrm{du}$ CJA et du CAG, mais que nous devrions encourager la croissance du CJA et du CAG par le truchement d'un soutien positif de ce que nous faisons. Quatrièmement, mes expériences à ces deux conférences ont soulevé la question des auditoires multiples. En particulier au FICCDAT, bon nombre de participants que j'ai rencontrés étaient des praticiens et des porte-parole. Ce sont aussi des groupes importants parmi ceux qui reçoivent le CJA et qui participent au CAG. Encore une fois dans le contexte du mandat du CJA, la question que je me pose et que je vous pose est: «Que puis-je faire en tant que rédacteur en chef pour atteindre de manière plus efficace les auditoires multiples de la revue et du CAG? ».

Dans le présent numéro du CJA, nous avons lancé une nouvelle section, "Notes sur les politiques et les pratiques (Policy and Practice Notes). Comme la section de longue date " Notes sur la recherche » (Research Notes) du CJA, les nouvelles « Notes sur les politiques et les pratiques » visent à encourager les auteurs notamment ceux qui ont conçu des outils d'évaluation, effectué des évaluations préliminaires des pratiques novatrices, qui sont axés sur les questions de politiques, et qui peuvent placer leur travail dans le contexte plus large de la recherche universitaire récente - de présenter leur travail au CJA. Les présentations de "Notes sur les politiques et pratiques » seront jugées par des pairs et elles doivent respecter les critères universitaires d'excellence que nous cherchons à instaurer dans chaque communication publiée dans le $C J A$, que ce soit un article, des notes de recherche ou, maintenant, une note sur les politiques ou les pratiques. Que votre principal intérêt réside dans les politiques et les pratiques ou dans n'importe lequel des autres secteurs de la gérontologie, je vous incite à lire la première « Note sur les politiques et les pratiques ", " Méthodologie d'enquête sur les chutes des aînés - Une approche systémique à l'étude des chutes chez les aînés » (Seniors Falls Investigative Methodology (SFIM)—A Systems Approach to the Study of Fall in Seniors) dans le présent numéro et à me laisser savoir si vous êtes d'accord avec la nouvelle orientation $\mathrm{du} C J A$, à mesure que nous tentons d'atteindre tous nos auditoires.

N'hésitez pas à communiquer avec moi si vous avez des idées à partager sur cette nouvelle initiative ou sur tout autre aspect de la revue. Vous pouvez me rejoindre à mark.rosenberg@queensu.ca

Mark Rosenberg

Rédacteur en chef 